\title{
A Note on Parabolic Liouville Theorems and Blow-Up Rates for a Higher-Order Semilinear Parabolic System
}

\author{
Guocai Cai, ${ }^{1}$ Hongjing Pan, ${ }^{2}$ and Ruixiang $X$ ing ${ }^{3}$ \\ ${ }^{1}$ School of Mathematical Sciences, Xiamen University, Xiamen 361005, China \\ 2 School of Mathematical Sciences, South China Normal University, Guangzhou 510631, China \\ ${ }^{3}$ School of Mathematics \& Computational Science, Sun Yat-sen University, Guangzhou 510275, China
}

Correspondence should be addressed to Ruixiang Xing, xingrx@mail.sysu.edu.cn

Received 30 May 2011; Accepted 7 September 2011

Academic Editor: Sining Zheng

Copyright (c) 2011 Guocai Cai et al. This is an open access article distributed under the Creative Commons Attribution License, which permits unrestricted use, distribution, and reproduction in any medium, provided the original work is properly cited.

We improve some results of Pan and Xing (Journal of Mathematical Analysis and Applications, vol. 339 , no. 1, pp. 248-258, 2008) and extend the exponent range in Liouville-type theorems for some parabolic systems of inequalities with the time variable on $\mathbb{R}$. As an immediate application of the parabolic Liouville-type theorems, the range of the exponent in blow-up rates for the corresponding systems is also improved.

\section{Introduction}

In this paper, we are concerned with the following two problems: one is blow-up rates for blow-up solutions of the higher-order semilinear parabolic system

$$
\begin{gathered}
u_{t}+(-\Delta)^{m} u=|v|^{p}, \quad v_{t}+(-\Delta)^{m} v=|u|^{q}, \quad(x, t) \in \mathbb{R}^{N} \times(0, T), \\
u(x, 0)=u_{0}(x) \in L^{\infty}\left(\mathbb{R}^{N}\right), \quad v(x, 0)=v_{0}(x) \in L^{\infty}\left(\mathbb{R}^{N}\right),
\end{gathered}
$$

where $m \geqslant 1$ and $p, q>1$; the other is parabolic Liouville theorems for the problem

$$
\begin{gathered}
u_{t}+(-\Delta)^{m} u=|v|^{p}, \quad v_{t}+(-\Delta)^{m} v=|u|^{q}, \quad(x, t) \in \mathbb{R}^{N} \times \mathbb{R}, \\
u(x, t) \in L_{\mathrm{loc}}^{p}\left(\mathbb{R}^{N+1}\right), \quad v(x, t) \in L_{\mathrm{loc}}^{q}\left(\mathbb{R}^{N+1}\right),
\end{gathered}
$$


where $m \geqslant 1$ and $p, q>1$. The first problem is directly related to the second one. Actually, blow-up rates of the blow-up solutions, by scaling arguments, are often converted to nonexistence of solutions of some limiting problems with $t \in \mathbb{R}$ (see, e.g., Poláčik and Quittner [1] and Xing [2]).

Recall that, in his famous paper [3], Fujita studied the initial value problem

$$
\begin{gathered}
u_{t}-\Delta u=u^{p}, \quad(x, t) \in \mathbb{R}^{N} \times(0, \infty), \\
u(x, 0)=u_{0}(x), \quad x \in \mathbb{R}^{N},
\end{gathered}
$$

for nonnegative initial data $u_{0}$. He obtained the following.

(i) If $1<p<1+2 / N$, then the only nonnegative global solution is $u \equiv 0$.

(ii) If $p>1+2 / N$, then there exist global solutions for some small initial value.

The number $1+2 / N$ belonging to Case (i) had been answered in [4-7], and an elegant proof was given by Weissler [7]. The number $1+2 / N$ is named the critical blow-up exponent (or critical Fujita exponent).

Ever since then, Fujita's result has been given great attention and extended in various directions. One direction is to consider the problems on other domains. For example, $\mathbb{R}^{N}$ is replaced by a cone or exterior of a bounded domain, and so forth. Another direction is to extend these results to more general equations and systems (see the survey papers [8-10] and references therein). We just briefly describe the results directly connected to our problems.

(1) The systems from the point of view of the critical blow-up exponent originate in the 1990s. Escobedo and Herrero [11] discussed the following weakly coupled secondorder parabolic system

$$
\begin{gathered}
u_{t}-\Delta u=v^{p}, \quad v_{t}-\Delta v=u^{q}, \quad(x, t) \in \mathbb{R}^{N} \times(0, T), \\
u(x, 0)=u_{0}(x) \geqslant 0 \in L^{\infty}\left(\mathbb{R}^{N}\right), \quad v(x, 0)=v_{0}(x) \geqslant 0 \in L^{\infty}\left(\mathbb{R}^{N}\right),
\end{gathered}
$$

with $p>0$ and $q>0$. They established the following.

(i) If $0<p q \leqslant 1$, then all solutions are global.

(ii) If $p q>1$ and $N / 2 \leqslant \max \{(p+1) /(p q-1),(q+1) /(p q-1)\}$, then every nontrivial solution blows up in finite time.

(iii) If $p q>1$ and $N / 2>\max \{(p+1) /(p q-1),(q+1) /(p q-1)\}$, then there exist both global solutions and blow-up solutions.

(2) Egorov et al. [12] considered a class of higher-order parabolic system of inequalities and gave some results about nonexistence of the nontrivial global solutions with initial data having nonnegative average value.

(3) A natural generalization of classical weakly coupled system (1.4) are the higherorder parabolic system (1.1). Pang et al. [13] studied (1.1) and obtained the following results.

(i) If $N / 2 m \leqslant \min \{(p+1) /(p q-1),(q+1) /(p q-1)\}$, then every solution with initial data having positive average value does not exist globally in time. 
(ii) If $N / 2 m>\max \{(p+1) /(p q-1),(q+1) /(p q-1)\}$, then global solutions with small initial data exist.

Notice that there exists a gap between the range of exponent in the two cases. In fact, in an earlier monograph [14], Mitidieri and Pokhozhaev have shown that Case (i) holds true for $N / 2 m \leqslant \max \{(p+1) /(p q-1),(q+1) /(p q-1)\}$ (see [14, Example 38.2]). Integrating these results in $[13,14]$, one directly obtains a complete Fujita-type theorem for the higher-order parabolic system (1.1).

Theorem 1.1. Assume $p>1$ and $q>1$. Then

(i) if $N / 2 m \leqslant \max \{(p+1) /(p q-1),(q+1) /(p q-1)\}$, then every solution of (1.1) with initial data having positive average value does not exist globally in time;

(ii) if $N / 2 m>\max \{(p+1) /(p q-1),(q+1) /(p q-1)\}$, then global solutions of $(1.1)$ with small initial data exist.

(4) Recently, Pan and Xing [15] considered the problem (1.2) and proved a parabolic Liouville theorem; that is, if $N / 2 m \leqslant \min \{(q+1) /(p q-1),(p+1) /(p q-1)\}$, then the global solution of (1.2) is trivial. As an immediate application of the result, blow-up rates for the problem (1.1) is also obtained:

Let $(u, v)$ be a solution of (1.1) which blows up at a finite time $T$. Then there is a constant $C>0$ such that $\sup _{x \in \mathbb{R}^{N}}|u(x, t)|, \sup _{x \in \mathbb{R}^{N}}|v(x, t)| \leqslant C(T-t)^{-(q+1) /(p q-1)}$ for $N / 2 m \leqslant$ $\min \{(p+1) /(p q-1),(q+1) /(p q-1)\}$.

The purpose of this note is to improve the results of [15]. More precisely, we will extend the exponent range from $N / 2 m \leqslant \min \{(q+1) /(p q-1),(p+1) /(p q-1)\}$ to $N / 2 m \leqslant$ $\max \{(q+1) /(p q-1),(p+1) /(p q-1)\}$ for both blow-up rates and parabolic Liouville theorems of [15]. The main results of this paper are Theorems 2.1 and 3.1. Our methods are similar to $[14,15]$. In fact, the present Theorem 2.1 will be proved by modifying part of the proof of Theorem 4.3 of [15].

The organization of this paper is as follows. In Section 2, we improve the range of the exponent for parabolic Liouville-type theorems in [15]. As a direct application, the exponent range in blow-up rates for corresponding systems is extended in Section 3.

\section{Parabolic Liouville-Type Theorem for Higher-Order System of Inequalities}

In this section, we will improve the exponent range of some parabolic Liouville-type theorems for higher-order semilinear parabolic systems.

Now we consider a class of more general parabolic systems of inequalities than (1.2). Let $L=L\left(t, x, D_{x}\right)$ be a differential operator of order $\ell$ :

$$
L[v]:=\sum_{|\alpha|=\ell} D^{\alpha}\left(a_{\alpha}(t, x, v) v\right)
$$

and let $M$ be a differential operator of order $h$ :

$$
M[v]:=\sum_{|\beta|=h} D^{\beta}\left(b_{\beta}(t, x, v) v\right),
$$

where $a_{\alpha}(t, x, v)$ and $b_{\beta}(t, x, v)$ are bounded functions defined for $t \in \mathbb{R}, x \in \mathbb{R}^{N}$, and $v \in \mathbb{R}$. 
Consider the set of $(u, v)$ satisfying the inequalities:

$$
\frac{\partial u}{\partial t} \geqslant L[u]+|v|^{q_{2}}, \quad \frac{\partial v}{\partial t} \geqslant M[v]+|u|^{q_{1}}, \quad(x, t) \in \mathbb{R}^{N} \times \mathbb{R},
$$

in the following weak sense: if $\psi \in C_{0}^{\max \{\ell, h\}}\left(\mathbb{R}^{N+1}\right)$ and $\psi(x, t) \geqslant 0$, then

$$
\begin{aligned}
& -\int \frac{\partial \psi}{\partial t} u d x d t-\int u L^{*}[\psi] d x d t \geqslant \int|v|^{q_{2}} \psi d x d t \\
& -\int \frac{\partial \psi}{\partial t} v d x d t-\int v M^{*}[\psi] d x d t \geqslant \int|u|^{q_{1}} \psi d x d t
\end{aligned}
$$

Here and in the following, if the limits of integration are not given, then the integrals are taken over the space $\mathbb{R}^{N} \times \mathbb{R}$, and

$$
L^{*}[\psi]:=\sum_{|\alpha|=\ell} a_{\alpha}(t, x, u)(-D)^{\alpha} \psi, \quad M^{*}[\psi]:=\sum_{|\alpha|=h} b_{\alpha}(t, x, u)(-D)^{\alpha} \psi
$$

Here is the main result of this section.

Theorem 2.1. If two functions $u(x, t) \in L_{\mathrm{loc}}^{q_{1}}\left(\mathbb{R}^{N+1}\right)$ and $v(x, t) \in L_{\mathrm{loc}}^{q_{2}}\left(\mathbb{R}^{N+1}\right)$ satisfy (2.4) and (2.5), then $u(x, t) \equiv 0, v(x, t) \equiv 0$ for $q_{1}, q_{2}>1$ and $\left(q_{1}, q_{2}\right) \in \Gamma_{1} \cup \Gamma_{2}$, where

$$
\begin{gathered}
\Gamma_{1}=\left\{\left(q_{1}, q_{2}\right) \mid \frac{N}{\min \{\ell, h\}} \leqslant \max \left\{\frac{q_{1}+1}{q_{1} q_{2}-1}, \frac{q_{2}+1}{q_{1} q_{2}-1}\right\}\right\}, \\
\Gamma_{2}=\left\{\left(q_{1}, q_{2}\right) \mid N+\max \{\ell, h\} \leqslant \max \left\{h+\frac{q_{1} \ell+h}{q_{1} q_{2}-1}, \ell+\frac{q_{2} h+\ell}{q_{1} q_{2}-1}\right\}\right\} .
\end{gathered}
$$

Remark 2.2. In fact, we will extend the range of the exponents $q_{1}, q_{2}$ in Theorem 4.3 of [15] from

$$
\left\{\left(q_{1}, q_{2}\right) \mid \frac{N}{\min \{\ell, h\}} \leqslant \min \left\{\frac{q_{1}+1}{q_{1} q_{2}-1}, \frac{q_{2}+1}{q_{1} q_{2}-1}\right\}\right\}
$$

to $\Gamma_{1} \cup \Gamma_{2}$. Obviously, $\Gamma_{1}$ contains the range of the exponent in [15].

As an immediate application, we take $L=M=-(-\Delta)^{m}, g_{1}(u, v)=|v|^{p}$, and $g_{2}(u, v)=$ $|u|^{q}$.

Corollary 2.3. If two functions $u(x, t) \in L_{\mathrm{loc}}^{p}\left(\mathbb{R}^{N+1}\right)$ and $v(x, t) \in L_{\mathrm{loc}}^{q}\left(\mathbb{R}^{N+1}\right)$ satisfy

$$
u_{t}+(-\Delta)^{m} u=|v|^{p}, \quad v_{t}+(-\Delta)^{m} v=|u|^{q},
$$


on $\mathbb{R}^{N} \times \mathbb{R}$, then $u(x, t) \equiv 0, v(x, t) \equiv 0$ for $p, q>1$ belonging to the following set:

$$
\left\{(p, q) \mid \frac{N}{2 m} \leqslant \max \left\{\frac{q+1}{p q-1}, \frac{p+1}{p q-1}\right\}\right\}
$$

Remark 2.4. In fact, the present Theorem 2.1 will be proved by modifying part of the proof of Theorem 4.3 of [15]. In the following proof, the part before the inequalities (2.24) is the same as that in Theorem 4.3 of [15]. The main difference between the proofs is the discussion of the four cases in the last part of the proof. For completeness of arguments as well as convenience of readers, we give a detailed proof of the theorem.

Proof of Theorem 2.1. Let $\phi \in C_{0}^{\max \{\ell, h\}}(\mathbb{R}), \phi \geqslant 0$, and

$$
\phi(s)= \begin{cases}1 & \text { as } s \leqslant 1 \\ 0 & \text { as } s \geqslant 2\end{cases}
$$

Suppose that there exists a positive constant $C$ such that

$$
\begin{array}{ll}
\left|\phi^{\prime}\right|(s) \leqslant C \phi^{1 / q_{1}}(s), \quad\left|\phi^{(\ell)}\right|(s) \leqslant C \phi^{1 / q_{1}}(s), \\
\left|\phi^{\prime}\right|(s) \leqslant C \phi^{1 / q_{2}}(s), \quad\left|\phi^{(h)}\right|(s) \leqslant C \phi^{1 / q_{2}}(s) .
\end{array}
$$

In order to find such a function, one also assume that, for $3 / 2<s<2, \phi(s)=(2-s)^{\delta}$ with $\delta>\max \left\{\ell q_{1} /\left(q_{1}-1\right), h q_{2} /\left(q_{2}-1\right)\right\}$.

Let

$$
\psi_{R}(x, t)=\phi\left(\frac{|t|^{2}+|x|^{2 \sigma}}{R^{2 \sigma}}\right), \quad R>0,
$$

the value of the parameter $\sigma>0$ will be determined below. Now putting $\psi=\psi_{R}(x, t)$ in $(2.4)$ and (2.5) and letting

$$
I I=\int|u|^{q_{1}} \psi_{R} d x d t, I I I=\int|v|^{q_{2}} \psi_{R} d x d t
$$

we have

$$
\begin{aligned}
& I I I \leqslant-\int \frac{\partial \psi_{R}}{\partial t} u d x d t-\int u L^{*}\left[\psi_{R}\right] d x d t \\
& I I \leqslant-\int \frac{\partial \psi_{R}}{\partial t} v d x d t-\int v M^{*}\left[\psi_{R}\right] d x d t .
\end{aligned}
$$


The Hölder inequality implies

$$
\begin{aligned}
-\int \frac{\partial \psi_{R}}{\partial t} u d x d t \leqslant C_{0} \int_{\operatorname{supp} \frac{\partial \psi_{R}}{\partial t}}|u| \psi_{R}^{1 / q_{1}} \frac{|t|}{R^{2 \sigma}} d x d t \\
\leqslant C_{1}\left\{\int_{\operatorname{supp} \frac{\partial \psi_{R}}{\partial t}}|u|^{q_{1}} \psi_{R} d x d t\right\}^{1 / q_{1}} \cdot\left\{\int_{\operatorname{supp} \frac{\partial \psi_{R}}{\partial t}} \frac{1}{R^{\sigma q_{1}^{\prime}}} d x d t\right\}^{1 / q_{1}^{\prime}} \\
\leqslant C_{2}\left\{\int_{\operatorname{supp} \frac{\partial \psi_{R}}{\partial t}}|u|^{q_{1}} \psi_{R} d x d t\right\}^{1 / q_{1}} \cdot\left(R^{N+\sigma-\sigma q_{1} /\left(q_{1}-1\right)}\right)^{\left(q_{1}-1\right) / q_{1}}, \\
-\int u L^{*}\left[\psi_{R}\right] d x d t \leqslant C_{3}\left\{\int_{\Upsilon_{1}}|u|^{q_{1}} \psi_{R} d x d t\right\}^{1 / q_{1}} \cdot\left(R^{N+\sigma-\ell q_{1} /\left(q_{1}-1\right)}\right)^{\left(q_{1}-1\right) / q_{1}},
\end{aligned}
$$

where $\Upsilon_{1}=\left\{(x, t): t \in \mathbb{R}, D_{x}^{\alpha} \psi_{R}(x, t) \neq 0\right.$ for some $\left.\alpha\right\}$ and $q_{1}^{\prime}=q_{1} /\left(q_{1}-1\right)$. It is essential here that the operator $L^{*}$ contains the derivatives of order $\ell$ only. It is obvious that (supp $\left.\frac{\partial \psi_{R}}{\partial t} \cup \Upsilon_{1}\right) \subset$ $\Sigma \triangleq\left\{(x, t): t \in \mathbb{R},|t|^{2}+|x|^{2 \sigma}>R^{2 \sigma}\right\}$, and therefore inequality (2.15) implies that

$$
\begin{aligned}
I I I & \leqslant C_{4}\left\{\int_{\Sigma}|u|^{q_{1}} \psi_{R} d x d t\right\}^{1 / q_{1}} \cdot R^{(N+\sigma)\left(q_{1}-1\right) / q_{1}}\left(R^{-\sigma}+R^{-\ell}\right) \\
& \leqslant C_{4}\left\{\int_{\Sigma}|u|^{q_{1}} \psi_{R} d x d t\right\}^{1 / q_{1}} R^{A} \\
& \leqslant C_{4} I I^{1 / q_{1}} R^{A}
\end{aligned}
$$

with $A=(N+\sigma)\left(q_{1}-1\right) / q_{1}-\min \{\sigma, \ell\}$. Similarly,

$$
\begin{aligned}
I I & \leqslant C_{5}\left\{\int_{\Sigma}|v|^{q_{2}} \psi_{R} d x d t\right\}^{1 / q_{2}} R^{(N+\sigma)\left(q_{2}-1\right) / q_{2}}\left(R^{-\sigma}+R^{-h}\right) \\
& \leqslant C_{5}\left\{\int_{\Sigma}|v|^{q_{2}} \psi_{R} d x d t\right\}^{1 / q_{2}} R^{B} \\
& \leqslant C_{5} I I I^{1 / q_{2}} R^{B}
\end{aligned}
$$


with $B=(N+\sigma)\left(q_{2}-1\right) / q_{2}-\min \{\sigma, h\}$. Then (2.20) and (2.23) lead to

$$
I I^{\left(q_{1} q_{2}-1\right) / q_{1} q_{2}} \leqslant C_{6} R^{B+A 1 / q_{2}}, \quad I I I^{\left(q_{1} q_{2}-1\right) / q_{1} q_{2}} \leqslant C_{7} R^{A+B 1 / q_{1}} .
$$

We consider the following cases.

Case 1. $B+A\left(1 / q_{2}\right)<0$. Let $R \rightarrow+\infty$ in the first inequality of (2.24), we obtain

$$
\int|u|^{q_{1}} d x d t=0
$$

which implies $u \equiv 0$. Combining with inequality (2.20) or equality (2.4), we get that $\int|v|^{q_{2}} d x d t=0$. Then $v \equiv 0$.

Case 2. $A+B\left(1 / q_{1}\right)<0$. Inequality (2.24) implies $v \equiv 0$. The inequality (2.23) or equality (2.5) leads that $u \equiv 0$.

Case 3. $B+A\left(1 / q_{2}\right)=0$. By (2.22) and (2.19), we have

$$
I I \leqslant C_{8}\left\{\int_{\Sigma}|u|^{q_{1}} \psi_{R} d x d t\right\}^{1 / q_{1} q_{2}} R^{B+A 1 / q_{2}}=C_{8}\left\{\int_{\Sigma}|u|^{q_{1}} \psi_{R} d x d t\right\}^{1 / q_{1} q_{2}} .
$$

And, from (2.24), we obtain that $\int|u|^{q_{1}} d x d t$ converge. Then, as $R \rightarrow+\infty$,

$$
\int_{\Sigma}|u|^{q_{1}} d x d t \longrightarrow 0
$$

Then $u \equiv 0$ and (2.20) implies $v \equiv 0$.

Case 4. $A+B\left(1 / q_{1}\right)=0$. Similarly to Case 3, the second inequality of (2.24) implies $\int|v|^{q} d x d t$ converging. $v \equiv 0$ follows from (2.19) and (2.22). Then by (2.23) or (2.5), $u \equiv 0$.

Taking $\sigma=\min \{\ell, h\}$, Cases 1 and $3: B+A\left(1 / q_{2}\right) \leqslant 0$ is equivalent to $N / \min \{\ell, h\} \leqslant$ $\left(q_{1}+1\right) /\left(q_{1} q_{2}-1\right)$, Cases 2 and $4: A+B\left(1 / q_{1}\right) \leqslant 0$ is $N / \min \{\ell, h\} \leqslant\left(q_{2}+1\right) /\left(q_{1} q_{2}-1\right)$. So the union of Cases $1-4$ is just the set $\Gamma_{1}$.

Similarly, taking $\sigma=\max \{\ell, h\}$, we obtain that the union of Cases $1-4$ is equivalent to the set $\Gamma_{2}$. Then we get the result.

\section{Blow-Up Rate Estimates for Parabolic Systems}

As an immediate application of Corollary 2.3, the range of the exponents $p, q$ in blow-up rates for the system (1.1) is also extended. We have the following theorem. 
Theorem 3.1. Let $(u, v)$ be a solution of (1.1) which blows up at a finite time T. Then there is a constant $C>0$ such that

$$
\sup _{x \in \mathbb{R}^{N}}|u(x, t)| \leqslant C(T-t)^{-(p+1) /(p q-1)}, \quad \sup _{x \in \mathbb{R}^{N}}|v(x, t)| \leqslant C(T-t)^{-(q+1) /(p q-1)}
$$

for

$$
\frac{N}{2 m} \leqslant \max \left\{\frac{p+1}{p q-1}, \frac{q+1}{p q-1}\right\}
$$

Since the proof of Theorem 3.1 is completely similar to Theorem 3.1 in [15], we omit it. Refer to [15] for all the details.

\section{Acknowledgment}

The first author was supported by Fundamental Research Funds for the Central Universities (Grant no. 2010121006). The second author was supported by NSFC (Grant no. 10901059). The third author was supported by NSFC (Grant nos. 10821067 and 11001277), RFDP (Grant no. 200805581023), Research Fund for the Doctoral Program of Guangdong Province of China (Grant no. 9451027501002416), and Fundamental Research Funds for the Central Universities.

\section{References}

[1] P. Poláčik and P. Quittner, "Liouville type theorems and complete blow-up for indefinite superlinear parabolic equations," in Nonlinear Elliptic and Parabolic Problems, vol. 64 of Progress in Nonlinear Differential Equations and Their Applications, pp. 391-402, Birkhäuser, Basel, The Switzerland, 2005.

[2] R. Xing, "The blow-up rate for positive solutions of indefinite parabolic problems and related Liouville type theorems," Acta Mathematica Sinica (English Series), vol. 25, no. 3, pp. 503-518, 2009.

[3] H. Fujita, "On the blowing up of solutions of the Cauchy problem for $u_{t}=\Delta u+u^{1+\alpha}$," Journal of the Faculty of Science. University of Tokyo. Section IA, vol. 13, pp. 109-124, 1966.

[4] D. G. Aronson and H. F. Weinberger, "Multidimensional nonlinear diffusion arising in population genetics," Advances in Mathematics, vol. 30, no. 1, pp. 33-76, 1978.

[5] K. Hayakawa, "On nonexistence of global solutions of some semilinear parabolic differential equations," Proceedings of the Japan Academy, vol. 49, pp. 503-505, 1973.

[6] K. Kobayashi, T. Sirao, and H. Tanaka, "On the growing up problem for semilinear heat equations," Journal of the Mathematical Society of Japan, vol. 29, no. 3, pp. 407-424, 1977.

[7] F. B. Weissler, "Existence and nonexistence of global solutions for a semilinear heat equation," Israel Journal of Mathematics, vol. 38, no. 1-2, pp. 29-40, 1981.

[8] K. Deng and H. A. Levine, "The role of critical exponents in blow-up theorems: the sequel," Journal of Mathematical Analysis and Applications, vol. 243, no. 1, pp. 85-126, 2000.

[9] V. A. Galaktionov and J. L. Vázquez, "The problem of blow-up in nonlinear parabolic equations," Discrete and Continuous Dynamical Systems. Series A, vol. 8, no. 2, pp. 399-433, 2002.

[10] H. A. Levine, "The role of critical exponents in blowup theorems," SIAM Review, vol. 32, no. 2, pp. 262-288, 1990.

[11] M. Escobedo and M. A. Herrero, "Boundedness and blow up for a semilinear reaction-diffusion system," Journal of Differential Equations, vol. 89, no. 1, pp. 176-202, 1991. 
[12] Y. V. Egorov, V. A. Galaktionov, V. A. Kondratiev, and S. I. Pohozaev, "On the necessary conditions of global existence to a quasilinear inequality in the half-space," Comptes Rendus de l'Académie des Sciences. Série I, vol. 330, no. 2, pp. 93-98, 2000.

[13] P. Y. H. Pang, F. Sun, and M. Wang, "Existence and non-existence of global solutions for a higher-order semilinear parabolic system," Indiana University Mathematics Journal, vol. 55, no. 3, pp. 1113-1134, 2006.

[14] È. Mitidieri and S. I. Pokhozhaev, “A priori estimates and the absence of solutions of nonlinear partial differential equations and inequalities," Trudy Matematicheskogo Instituta Imeni V. A. Steklova, vol. 234, pp. 1-384, 2001.

[15] H. Pan and R. Xing, "Blow-up rates for higher-order semilinear parabolic equations and systems and some Fujita-type theorems," Journal of Mathematical Analysis and Applications, vol. 339, no. 1, pp. 248258, 2008. 


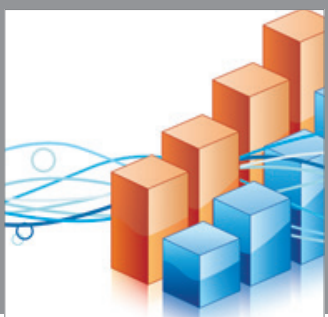

Advances in

Operations Research



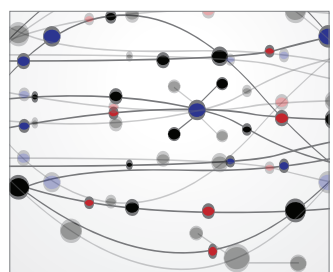

\section{The Scientific} World Journal
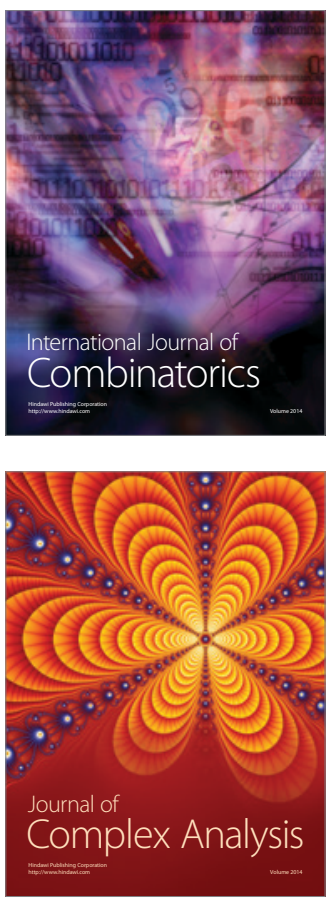

International Journal of

Mathematics and

Mathematical

Sciences


Journal of

Applied Mathematics
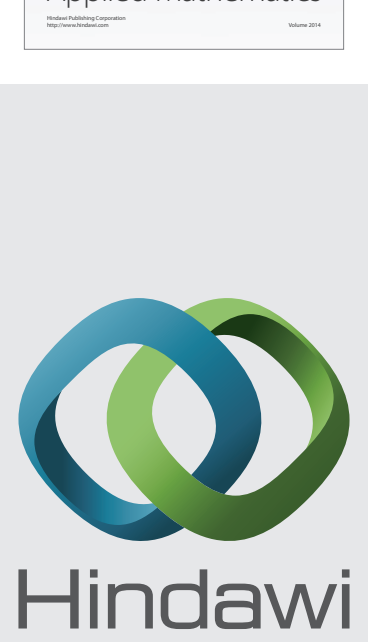

Submit your manuscripts at http://www.hindawi.com
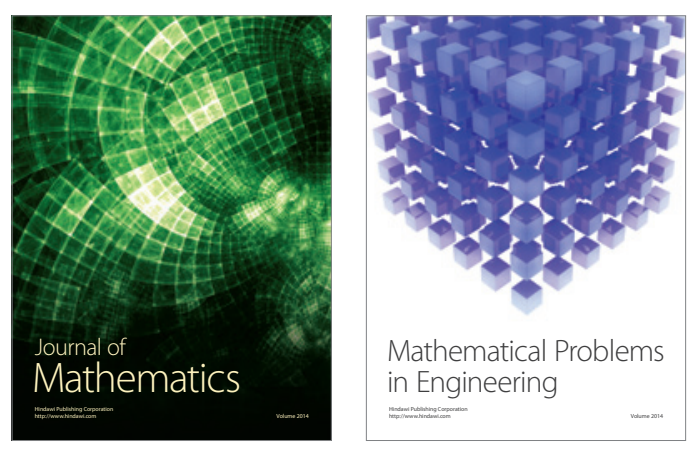

Mathematical Problems in Engineering
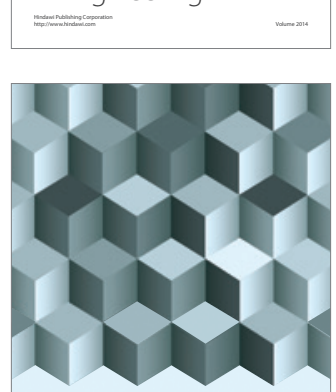

Journal of

Function Spaces
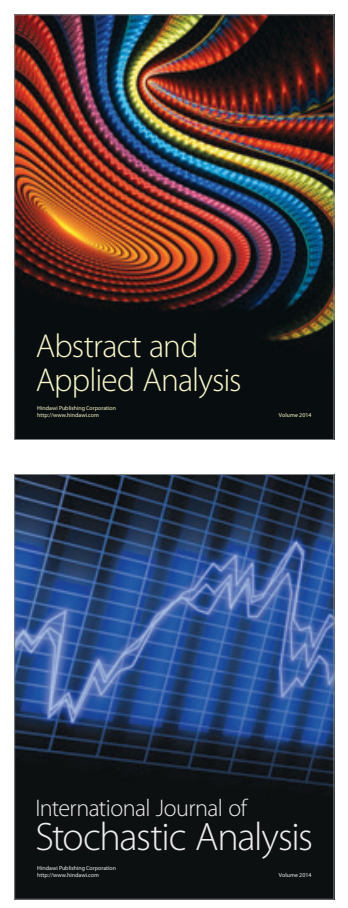

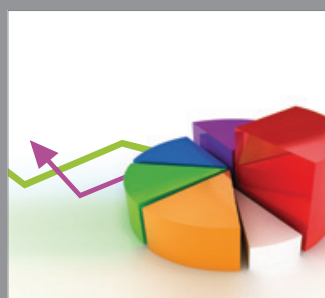

ournal of

Probability and Statistics

Promensencen
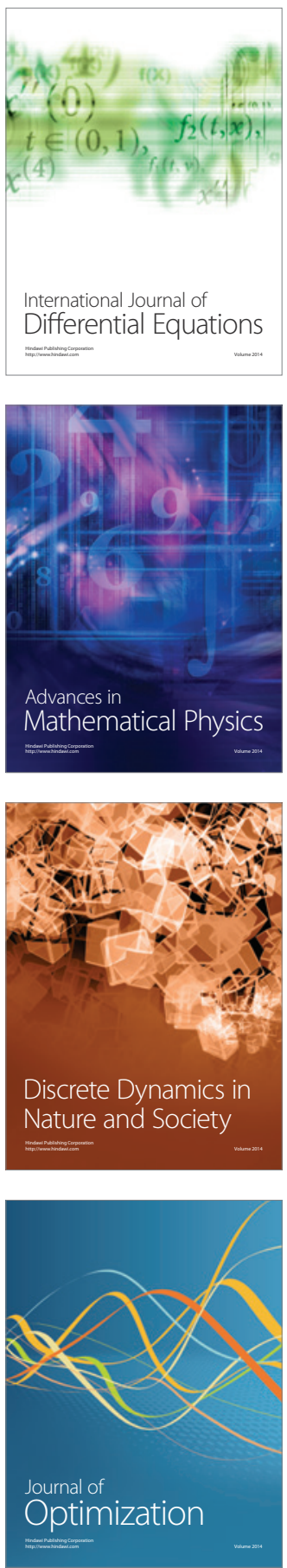